\title{
Central pontine myelinolysis in a patient with persistent mild hypernatremia following cadaver donor liver transplantation
}

\author{
Sang Hoon Yoon, Ji-Yong Park, Sung-Uk Choi, Seung Zhoo Yoon, and Hye Won Lee \\ Department of Anesthesiology and Pain Medicine, College of Medicine, Korea University, Seoul, Korea
}

We experienced a case of central pontine myelinolysis (CPM) development in the setting of mild hypernatremia. A 46-yearold female was diagnosed with cryptogenic liver cirrhosis due to autoimmune disease. She was scheduled for emergency cadaver donor liver transplantation. A pre-operative examination revealed the following parameters: total bilirubin $(36.22 \mathrm{mg} /$ $\mathrm{dl})$, aspartate aminotransferase (52 IU/L), alanine transaminase (49 IU/L), glutamate-pyruvate transaminase (101 IU/L), serum creatinine $(1.2 \mathrm{mg} / \mathrm{dl})$, serum sodium $(147 \mathrm{mmol} / \mathrm{L})$, serum magnesium (0.87 $\mathrm{mmol} / \mathrm{L})$, plasma osmotic pressure (289 $\mathrm{mOsm} / \mathrm{kg} \mathrm{H}_{2} \mathrm{O}$ ), Child-Pugh score C, MELD score (36). During the operation, the sodium concentration was maintained between 145 and $150 \mathrm{mmol} / \mathrm{L}$. The total operation time was $15 \mathrm{hr}$.

Electrolytes were immediately re-evaluated after the operation in the intensive care unit: serum sodium was $149 \mathrm{mmol} / \mathrm{L}$ and plasma osmotic pressure was $291 \mathrm{mOsm} / \mathrm{kg} \mathrm{H}_{2} \mathrm{O}$. At 19 hours following surgery, the patient was extubated. The patient was on hemofiltration due to degraded renal function and persisting hypernatremia. The patient was treated with tacrolimus $1 \mathrm{mg}$, mycophenolic acid $250 \mathrm{mg}$ (oral, twice daily), and prednisolone $5 \mathrm{mg}$ (iv).

During the first week, the patient was nearly alert and could provide correct answers to simple questions. Tendon reflexes were symmetric and active, with pathological sign (-). On day 7 after the surgery, the patient became lethargic, only opened eyes when powerfully stimulated, could not pronounce or perform autonomic limb activities, and thus underwent tracheostomy. She was mechanically ventilated due to a pulmonary infection. Since then, the patient's mental status continued to decline. A review of the patient's laboratory data showed that her hypernatremia continued to be between 150 and $157 \mathrm{mmol} / \mathrm{L}$ and plasma osmotic pressure was persisted between 280 and $300 \mathrm{mOsm} / \mathrm{kg}$ $\mathrm{H}_{2} \mathrm{O}$. After all our efforts for correcting serum sodium level and osmolarity using half saline and hemofiltration, hypernatremia persisted. On day 18 after the surgery, the patient developed a moderate coma, with good recovery of liver function. On day 21, brain magnetic resonance imaging revealed CPM (Fig. 1). Subsequently, the patient developed severe sepsis and multiple organ failure, and expired.

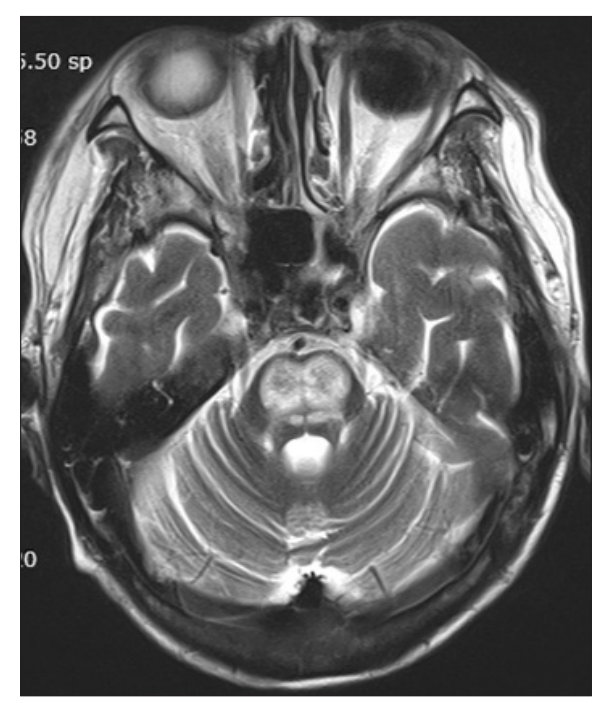

Fig. 1. Magnetic resonance imaging displayed high-signal intensity on T2-weighted images of the central pons $21 \mathrm{~d}$ after surgery.

Corresponding author: Seung Zhoo Yoon, M.D., Ph.D., Department of Anesthesiology and Pain Medicine, College of Medicine, Korea University, Anam-dong 5-ga, Seongbuk-gu, Seoul 136-705, Korea. Tel: 82-2-920-5632, Fax: 82-2-929-2936, E-mail: monday1031@yahoo.co.kr (c) This is an open-access article distributed under the terms of the Creative Commons Attribution Non-Commercial License (http:// creativecommons.org/licenses/by-nc/3.0/), which permits unrestricted non-commercial use, distribution, and reproduction in any medium, provided the original work is properly cited. 
Our patient differs from the classic cases of CPM as hyponatremia was not present. The unique feature of our case is that CPM developed in the setting of relatively mild hypernatremia. One may intuitively say that the authors should correct the hypernatremia on recognition. However, once developed, it is known that overly rapid correction of hypernatremia is potentially very dangerous, and can lead to cerebral edema, potentially resulting in seizures, permanent brain damage, or death [1]. In addition, Go et al. [2] reported a case of CPM and EPM after rapid correction of hypernatremia. The patient's initial serum sodium value of $186 \mathrm{mEq} / \mathrm{L}$ was corrected to 139 $\mathrm{mEq} / \mathrm{L}$ in 5 days.

Although rapid correction of hypernatremia has been reported in association with CPM, to our knowledge, where the serum sodium went from upper normal to hypernatremic, appears to be unique. In addition, the post-operative increase of sodium concentration never exceeded 1-2 mmol/L per day, yet the patient developed CPM. Several reports have shown that underlying hypokalemia may predispose patients to more pronounced myelin damage. In this patient, although the hypokalemia was mild, we cannot deny the possibility that it may have had a pathological effect with regard to the unusual accompaniment of CPM. Also, the post-operative lab results showed that the level of phosphates was normal.

The authors need to point out the recent conflicting animal experiments that have shown an alternative approach to prevent osmotic demyelination to re-lower serum sodium following overly rapid correction. Kengne et al. [3] reported that the relowering of the serum sodium after rapid correction of chronic hyponatremia was beneficial if performed early in the course (12 to $24 \mathrm{~h}$ ). The results suggest that after inadvertent rapid correction of hyponatremia, treatment options should favor re-lowering serum sodium. The increased permeability of the blood-brain barrier seen in osmotic demyelination syndrome may not be a primary pathophysiologic insult of CPM.

There was a limitation for our case in that we used Taclolimus for the postoperative immunosuppressive regimen. Fukazawa et al. [4] provided evidence of Tacrolimus-associated CPM after transplantation, which presented with a classic "lock-in syndrome" with radiographic confirmation.

In conclusion, our case illustrated the development of severe CPM in a patient with mild hypernatremia despite the absence regarding the rapid elevation of serum sodium and significant metabolic derangement. Slow correction of chronic hyponatremia or, in the case of our patient, avoidance of postoperative hypernatremia in the setting of an orthotopic liver transplant, is crucial for the prevention of central pontine myelinolysis. Our case may broaden the clinical spectrum of CPM and suggests that this clinical entity should be considered in various clinical settings.

\section{References}

1. Adrogue HJ, Madias NE. Hypernatremia. N Engl J Med 2000; 342: 1493-9.

2. Go M, Amino A, Shindo K, Tsunoda S, Shiozawa Z. A case of central pontine myelinolysis and extrapontine myelinolysis during rapid correction of hypernatremia. Rinsho Shinkeigaku 1994; 34: 1130-5.

3. Gankam Kengne F, Soupart A, Pochet R, Brion JP, Decaux G. Re-induction of hyponatremia after rapid overcorrection of hyponatremia reduces mortality in rats. Kidney Int 2009; 76: 614-21.

4. Fukazawa K, Nishida S, Aguina L, Pretto E Jr. Central pontine myelinolysis (CPM) associated with tacrolimus (FK506) after liver transplantation. Ann Transplant 2011; 16: 139-42. 